\title{
Neue Wachstumskurven für die Schweiz
}

\author{
Braegger, C ; Jenni, O G ; Konrad, D ; Molinari, L
}

Posted at the Zurich Open Repository and Archive, University of Zurich ZORA URL: https://doi.org/10.5167/uzh-55244

Journal Article

Published Version

Originally published at:

Braegger, C; Jenni, O G; Konrad, D; Molinari, L (2011). Neue Wachstumskurven für die Schweiz. Schweizerische Ärztezeitung, 92(7):229-230. 


\section{Neue Wachstumskurven für die Schweiz}

\author{
Christian Braeggera, \\ Oskar Jenni ${ }^{b}$, Daniel Konrad ${ }^{\text {, }}$ \\ Luciano Molinari ${ }^{b}$
}

Arbeitsgruppe Wachstumskurven des Kinderspitals Zürich (in alphabetischer Reihenfolge)

a Abteilung Gastroenterologie und Ernährung

b Abteilung Entwicklungspädiatrie

c Abteilung Endokrinologie/ Diabetologie

\section{* Die Literaturangaben finden sich im Internet unter www.saez.ch $\rightarrow$ Aktuelle Nummer oder $\rightarrow$ Archiv $\rightarrow 2011 \rightarrow 7$}

Seit über 30 Jahren wurden in der Schweiz die Perzentilenkurven der 1. Zürcher Longitudinalstudie (1. ZLS) zur Beurteilung des Wachstums gebraucht. Die 1. ZLS umfasste 274 gesunde Kinder (je 137 Knaben und Mädchen) mit den Geburtsjahrgängen 1954 bis 1956. Die Besonderheit dieser Studie lag darin, dass die einzelnen Kinder und ihre Wachstumsverläufe detailliert dokumentiert und beschrieben wurden $[1,2]^{*}$. Dazu wurden mehr als 20 anthropometrische Masse und das Knochenalter in mindestens jährlichen Abständen erfasst. Die 1. ZLS erlaubte Aussagen zur Stabilität des kindlichen Wachstums, prognostischen Aussagekraft von Körpermassen, Beziehung zwischen anthropometrischen Massen untereinander, Beziehung des Wachstums zwischen Eltern und Kind sowie der Entwicklung von Knochenreifung und Pubertätsmerkmalen.

\section{Warum braucht es neue Wachstumskurven?} Obwohl mit der 1. ZLS die weltweit wohl umfassendste Datenbank für das kindliche Wachstum zur Verfügung steht [3], gibt es mehrere Gründe für eine Überarbeitung der aktuellen Perzentilen, die in Praxen und Spitälern gebraucht werden.

\section{Verändertes Stillverhalten}

Bereits 1995 machte ein Fachausschuss der WHO darauf aufmerksam, dass Wachstum und Gewichtszunahme von gestillten Kindern mit den gängigen Wachstumskurven nicht zuverlässig erfasst werden können [4]. Tatsächlich belegen verschiedene Studien, dass gestillte Säuglinge zwar in den ersten Lebenswochen schneller wachsen und an Gewicht zunehmen als nicht gestillte Säuglinge, dass jedoch die gestillten Säuglinge nach wenigen Wochen eine deutlich langsamere Zunahme von Gewicht und Körperlänge zeigen [4]. Dies gilt auch für die Wachstumskurven der 1. ZLS.

Diese Beobachtung führte nicht selten zu unnötigen Abklärungen und gelegentlich sogar zur Empfehlung, gestillte und damit optimal ernährte Säuglinge zu früh zuzufüttern oder gar abzustillen. Die Ursache dafür liegt in der Tatsache, dass die meisten älteren Perzentilenkurven (so auch die Kurven der 1. ZLS) auf Daten von Kindern beruhen, die mehrheitlich nicht gestillt wurden. Aus diesem Grunde initiierte die WHO die Multicenter Growth Reference Study (MGRS), die auf einer Population von gesunden, optimal ernährten (das heisst gestillten) Kindern aus verschiedenen Kulturen beruht.

\section{Zusammenfassung}

Die Arbeitsgruppe Wachstumskurven des Kinderspitals Zürich hat in den letzten zwei Jahren neue Wachstumskurven erarbeitet. Die Kurven werden von der Schweizerischen Gesellschaft für Pädiatrie (SGP), der Ernährungskommission der SGP, der Arbeitsgruppe Adipositas der SGP, der Schweizerischen Gesellschaft für Pädiatrische Endokrinologie und Diabetologie (SGPED) sowie der Schweizerischen Gesellschaft für Entwicklungspädiatrie (SGEP) anerkannt. Die Kurvensets können von der Website des Kinderspitals Zürich und der Schweizerischen Gesellschaft für Pädiatrie als pdf-Files heruntergeladen werden. Gedruckte Versionen können im Schulungsund Wohnheim Rossfeld in Bern bezogen werden.

\section{Besondere Stichprobe der Zürcher Longitudinalstudien}

Das Studienziel der 1. ZLS war die Dokumentation von individuellen Wachstumsverläufen. Die Stichprobe ist mit je 137 Knaben und Mädchen für normative Referenzdaten zu klein. Zudem wurde in der 1. ZLS tendenziell eine Population mit Kindern überwiegend aus der Mittel- und Oberschicht aus der Region Zürich eingeschlossen. Die Studie ist damit nicht für die ganze Schweiz repräsentativ. In den letzten 15 Jahren hat sich zudem gezeigt, dass die Perzentilenkurven der 1. ZLS für das Gewicht wegen des starken Generationeneffektes nicht mehr zuverlässig sind. Die Kinder sind heute schwerer als noch vor 50 Jahren.

\section{Moderne statistische Methoden}

Die Wachstumskurven der 1. ZLS beruhen auf mit Spline Funktionen geglätteten empirischen Perzentilen. Diese Technik ist heute für die Berechnung von Perzentilenkurven nicht mehr zeitgemäss. State of the Art ist die LMS Methode von Tim Cole [5, 6], mit der die WHO-Wachstumskurven sowie die in diesem Artikel publizierten Kurven berechnet sind. Die mit dieser Methode berechneten Perzentilen sind in der Regel harmonisch. Die Methode nutzt die Daten durch eine Box-Cox Transformation zur Normalverteilung in statistisch effizienter Weise und erlaubt eine einfache Berechnung von Standard Deviation Scores (SDS, z- 
Scores), die wissenschaftlich und klinisch für die differenzierte Beurteilung von individuellen Verläufen notwendig sind.

\section{Unterschiedliche Perzentilen in der Deutsch- schweiz und der Romandie}

Die Perzentilenkurven der 1. ZLS wurden vorwiegend in der Deutschschweiz gebraucht. In der Romandie verwendete man meist die Kurven von Michel Sempé und Kollegen aus Paris, die wie die 1. ZLS aus den International Children's Center Studies stammen [7]. Ein Ersatz dieser zwei aktuell in der Schweiz verwendeten Kurvensätze durch die WHO-Kurven ermöglicht erstmals den Gebrauch einheitlicher Wachstumskurven in der gesamten Schweiz.

\section{Die neuen Wachstumskurven}

Die Abbildung 1 zeigt beispielhaft die Perzentilenkurven für Grösse, Gewicht und Kopfumfang von Mädchen vom 1. bis ins 18. Lebensjahr. Die Kurven zur Länge/Grösse stammen aus der WHO Multicenter Growth Reference Study (MGRS) [8, 9], in der zwischen 1997 und 2003 die Wachstumsdaten von 8500 optimal ernährten (das heisst gestillten) Kindern zwischen 0 und 5 Jahren aus verschiedenen Kontinenten und Ländern erhoben wurden (Brasilien, Ghana, Indien, Norwegen, Oman und USA) sowie aus der Rekonstruktion der National Center for Health Statistics (NCHS) Daten mit 22917 Kindern aus dem Jahre 1977 für die Alter 5-18 Jahre [10, 11]. Die Daten von 0-5 Jahren (MGRS) zeigen keine Differenzen zwischen den untersuchten Ländern und gelten darum auch für die Schweiz als zuverlässige Kurven.

Bedauerlicherweise wurden die NCHS Gewichtskurven von 1977 nur bis in das 10. Lebensjahr rekon-

\section{Abbildung 1}

Perzentilenkurven für Grösse, Gewicht und Kopfumfang von Mädchen vom 1. bis ins 18. Lebensjahr (Beispiel).

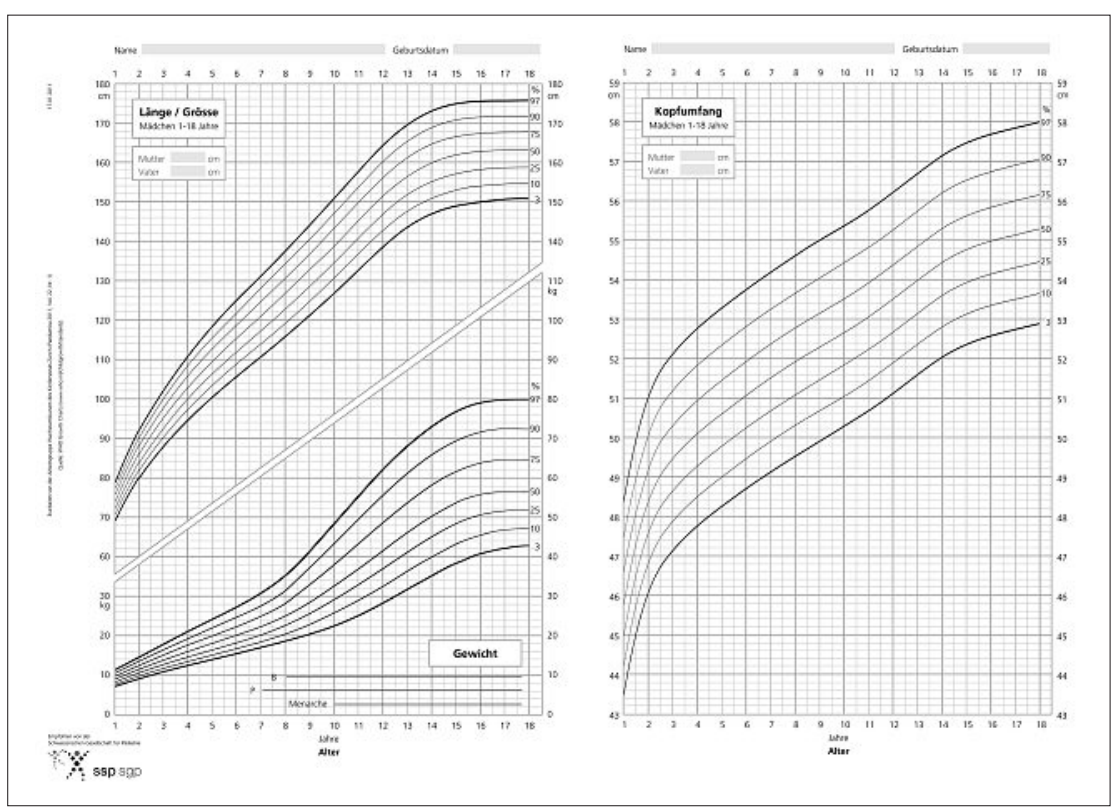

struiert [10, 11]. Die Arbeitsgruppe des Kinderspitals Zürich hat darum die Gewichtskurve mit den Daten der MGRS [9] und der NCHS [12] bis zum Alter von 18 Jahren neu berechnet (mit der LMS Methode von Cole). Die Kurven zum BMI stammen ebenfalls aus der WHO MGRS $[8,9]$ und der Rekonstruktion der NCHS Daten $[10,11]$.

In der MGRS wurden die Daten des Kopfumfangs nur bis zum Alter von 5 Jahren erhoben. Es gibt weltweit zahlreiche Studien über den Kopfumfang in den ersten 18 Lebensjahren, die sich erheblich voneinander unterscheiden. Die Arbeitsgruppe entschied sich darum, weiterhin die Schweizer Daten der Zürcher Longitudinalstudien zu verwenden. Die Kurven für den Kopfumfang beruhen auf den Daten der 2. Zürcher Longitudinalstudie (110 termingeborene Kinder, Geburtsjahrgänge 1974-1978) und der Zürcher Generationenstudie (Kinder der Probanden der 1. Studie, 320 Kinder, Geburtsjahrgänge 1974-1992).

Für die Beurteilung des Wachstums ist die Erfassung der Wachstumsgeschwindigkeit sehr wichtig. Perzentilenkurven für die Wachstumsgeschwindigkeit sind allerdings nicht einfach erhältlich, da diese auf longitudinal erhobenen Wachstumsdaten beruhen. Deshalb muss auch in Zukunft zur Beurteilung der Wachstumsgeschwindigkeit auf die 1. ZLS oder die Sempé-Kurven zurückgegriffen werden. Auch für weitere anthropometrische Masse ist die Originalpublikation von Prader und Kollegen nach wie vor eine wichtige Referenz [1].

Die Wachstumskurven von Früh- und Neugeborenen Kindern stammen aus der Publikation von Voigt und Kollegen [13]. Die Daten umfassen die Einlingsgeburten der Jahre 1995 bis 2000 aus der Bundesrepublik Deutschland (2,3 Mio. Neugeborene). Diese Perzentilenkurven gelten für die Beurteilung bei Geburt (Geburtsmasse) und sind nicht als Referenz für das postnatale Wachstum geeignet (siehe dazu [14]).

Die Kurvensets können von der Website des Kinderspitals Zürich (www.kispi.uzh.ch) und der SGP (www.swiss-pediatrics.org) als pdf-Files heruntergeladen werden. Gedruckte Versionen der Kurvensets können im Schulungs- und Wohnheim Rossfeld, Reichenbachstrasse 122, Postfach 699, 3004 Bern 4, buerozentrum@rossfeld.ch oder Fax 0313000299 bestellt werden. Die Kurven dürfen nicht ohne die Zustimmung der Arbeitsgruppe Wachstumskurven des Kinderspitals Zürich abgeändert werden.

\section{Dank}

Die Arbeitsgruppe dankt Monika Bloessner (WHO, Genf) für die wertvollen Hinweise zu den WHOKurven, Manfred Voigt (Institut für Perinatale Auxologie am Klinikum Südstadt in Rostock) für das Überlassen der Tabellen zum Neugeborenenkollektiv, Susanne Staubli (Kinderspital Zürich) für das Layout der Kurven und Felix H. Sennhauser (Kinderspital Zürich) für den Auftrag und die Unterstützung der Arbeitsgruppe. 


\section{Literatur}

1 Prader A, Largo RH, Molinari L, Issler C. Physical growth of Swiss children from birth to 20 years of age. First Zurich longitudinal study of growth and development. Helv Paediatr Acta Suppl. 1989; 52:1-125.

2 Gasser T, Kohler W, Muller HG, Kneip A, Largo R, Molinari L et al. Velocity and acceleration of height growth using kernel estimation. Ann Hum Biol. 1984;11(5):397-411.

3 Tanner JM. A brief history of the study of human growth. In: Ulijaszek SJ, Johnston FE, Preece MA, (eds). The Cambridge Encyclopedia of Human Growth and Development. Cambridge: Cambridge University Press; 1998.

4 WHO. An evaluation of infant growth: the use and interpretation of anthropometry in infants. Bulletin of the World Health Organization. 1995;73:165-74.

5 Cole TJ. Fitting smoothed centile curves to reference data. J R Stat Soc. 1988;151:385-418.

6 Cole TJ, Green PJ. Smoothing reference centile curves: the LMS method and penalized likelihood. Statistic Med. 1992;11:1305-19.

7 Sempé M, Pédron G, Roy-Pernot MP. Auxologie méthode et séquences. Paris: Théraplix; 1979.

8 WHO. The WHO Child Growth Standards. www.who.int/childgrowth/mgrs/eng. Accessed 12th december 2010.
9 De Onis M, Garza C, Victora CG, Onyango AW, Frongillo EA, Martines J. The WHO Multicentre Growth Reference Study. Food Nutr Bull. 2004; 25(1 Suppl):S3-84.

10 WHO. Growth reference data for 5-19 years. www. who.int/growthref/growthref_who_bull/en/index. html. Accessed 12th december 2010.

11 De Onis M, Onyango AW, Borghi E, Siyam A, Nishida C, Siekmann J. Development of a WHO growth reference for school-aged children and adolescents. Bull World Health Organ. 2007;85(9): 660-7.

12 Hamill PV, Drizd TA, Johnson CL, Reed RB, Roche AF. NCHS growth charts; 1976.

13 Voigt M, Fusch C, Olbertz D, Hartmann K, Rochow N, Renken C et al. Analyse des Neugeborenenkollektivs der Bundesrepublik Deutschland 12. Mitteilung: Vorstellung engmaschiger Perzentilwerte (-kurven) für die Körpermaße Neugeborener. Geburtsh Frauenheilk. 2006;66:956-70.

14 Bucher HU. Wie soll ein Übergewicht für Gestationsalter definiert werden? Paediatrica. 2010;21(5):42-4. 